\title{
Planetary exploration of Saturn moons Enceladus and Dione
}

\author{
J.R. Sanmartín ${ }^{\mathrm{a}, \mathrm{b}}$, J. Peláez ${ }^{\mathrm{b}, *}$ \\ ${ }^{a}$ Real Academia de ingenieria of Spain, Spain \\ - Universidad Politécnica de Madrid, Pz. G. Cisneros 3, Madrid, 28040, Spain
}

A R T ICLE INFO

\section{Keywords:}

Outer giants

lcy moons

Electrodynamic tethers

Magnetic capture

\begin{abstract}
A B S T R A C T
Search for habitability in Outer Planets moons, requires presence of proper chemistry, water, and energy. Dissipation from tidal forces is major energy source in evolution of Icy moon systems, generically exhibiting a multi-layer structure, with outer solid (ice) and intermediate liquid layers, and solid (rocky) core. Saturn moon Enceladus has been found to eject plumes of water vapour and ice, and very recently, hydrogen molecules, a tentative sign of chemistry supporting microbial life. Purpose of the present work is a preliminary design of a minor electrodynamic tether mission to visit Enceladus. Free tether capture and maneuvering would take the $\mathrm{S} / \mathrm{C}$ to a Satum orbit with periapsis very close to the planet and apoapsis at Enceladus orbit, the $S / C$ proved to be then in a 1:2 resonance with the moon, allowing parallel, conveniently slow flybys. Such mission could also involve visiting Dione, the $4^{\text {th }}$ largest Saturn moon, Enceladus and Dione being in a natural 1:2 Laplace resonance; it was recently brought to light that Dione exhibits multiple, parallel color lines, hundred kilometers long.
\end{abstract}

\section{Introduction}

Search for habitability in moons of Outer planets requires presence of sources of energy. Dissipation from tidal forces is a major energy source in evolution of Icy moon systems. Heating always accompanies dissipation but, in case of tidal heating, it will additionally present complex spatial distribution, reflecting on a multi-layer structure in the particular case of Icy moons. Subsurface oceans are a generic feature of large icy bodies at some point in their evolution. A standard interior model consists of an outer solid (ice) layer, an intermediate liquid layer, and a solid (rocky) core.

It was recently discovered that a Saturn moon, Enceladus, ejects plumes of water vapour and ice particles, evidence of a liquid water reservoit below the surface, in the South Polar Terrain [1]. More recently, in the Spring of 2017 , it was found that such plumes contain molecular hydrogen, considered sign of chemical reactions supporting microbial life [2,3]. Exploration of Enceladus would appear now of the greatest interest in the search for habitability in the Solar System.

As a moon rises a tide on its planet, they exchange angulat momentum, affecting satellite orbit and planet spin; the moon gains orbital energy at the expense of the planet rotational energy. In case of planets with several moons, a complex 3-body paradigm, Laplace resonance, involves two (or more) moons of a planet, forming by differential expansions of orbits due to tidal totques. At Jupiter, moons Io, Europa, and Ganymede are in 1:2:4 resonance, as established by P.S. Laplace at around the birth of thermodynamic science.

Enceladus is in a 1:2 resonance with the $4^{\text {th }}$ largest Saturn moon, Dione; Enceladus revolution and rotation periods are both 1.370 days, Dione's petiods being approximately 2.74 days. Dione itself became "moon of interest" very recently, in the Fall of 2018 , when it was brought to light a feature, observed in the course of the Cassini mission: Dione exhibits multiple color lines (linear virgae), like hundred kilometres long, parallel to the equator as latitudinal lines in a map [4]. It was suggested that such linear virgae are possibly due to impacts from dust-sized foreign material, with low mass and velocity to form streaks, while depositing onto the surface rather than forming craters.

A minor tether mission could allow exploring this Laplace resonance. Tethers can provide free propulsion and power for capture and then maneuvering, allowing frequent flyby-visits to selected moons, as suggested to explore Jovian moons [5]. Apoapsis lowering operation would follow capture, in equatorial orbit, by Satum [6]. As recalled in Sec. 2, tether Lorentz-drag is such that periapsis in the orbit of capture must be very close to the planet, making periapsis itself weakly affected by maneuvering to follow. In Sec. 3 it is shown that this makes quite effective exploration of Enceladus: a S/C with periapsis very close to Saturn and apoapsis at Enceladus orbit, is in 1:2 S/C-Enceladus resonance itself, allowing tangential, conveniently slow flybys of the moon. 
Following capture by Saturn, repeated Lorentz force around periapsis may bring the apoapsis first to an elliptical orbit at 1:1 resonance with Dione. After a number of flybys, with its Hollow Cathode off to explore Dione, as had been suggested for a minor tether mission to moon Europa [7], repeated tether operation might bring the apoapsis down to reach a resonance with Enceladus, for parallel flybys. Section 4 shows that Lorentz-drag peaking at periapsis greatly simplifies the analysis, and tether operation in touring Enceladus and Dione is explicitly determined. Results are discussed and conclusions summarized in Sec. 5.

\section{Saturn versus Neptune capture review}

Tether efficiency in spaceciaft-capture ( $S / C$-to-tether mass ratio, $M_{S C} / m_{t}$ ) goes down as $B^{2}$ for weak magnetic fields, because field $\mathbf{B}$ is twice involved: $B$ exerts Lorentz force per unit length $\mathbf{I} \wedge \mathbf{B}$ on tether current $\mathbf{I}$, actually driven by a motional electric field $\mathbf{E}_{m} \equiv \mathbf{v}^{\prime} \wedge \mathbf{B}$ in the $S / C$ reference frame, induced by the relative $S / C$ velocity $\mathbf{v}^{\prime}$ in the magnetized ambient plasma co-rotating with the planet. Capture is more effective the lower the incoming $S / C$ periapsis because the planetary dipole-field $\mathbf{B}$ decreases as inverse cube of distance to the planet, so Lorentz drag decreases rapidly, as the inverse $6^{\text {th }}$ power.

Also, since Lorentz-drag will peak at periapsis of a $S / C$ orbiting a planet, it will have little effect on periapsis itself. In the 2-body general relation between specific energy $\varepsilon$ and eccentricity $e$ at constant periapsis $r_{p}$,

$\varepsilon=(e-1) \mu / 2 r_{p}$

any sequence of drag-work steps will directly relate $e$ and $\varepsilon$. For a $S / C$ Hohmann-transfer between heliocentric circular orbits at Earth and planet, the arriving velocity $v_{\infty}$ of the hyperbolic orbit in the planetary frame provides an orbital specific energy $b_{t}=(1 / 2) v_{\infty}^{2}$. Using $v_{\infty}$ in (1) yields

$e_{h}-1=v_{\infty}^{2} R / \mu$

where $\mu$ is the gravitational constant and we set periapsis $r_{p} \approx R$. A Jovian gravity-assist [8] can reduce eccentricity, along with $v_{\infty}$, to a value $e_{h} \approx 1.01[6]$, helping magnetic capture of a $S / C$ into elliptical orbit.

Planetary capture, requiring negative Lorentz drag-work, $W_{d} / M_{s c}=\triangle \varepsilon<0$, allows writing

$\frac{\left|W_{d}\right|}{m_{t}}=\frac{\left|w_{d}\right|}{M_{S C}} \times \frac{M_{S C}}{m_{t}}=|\triangle \varepsilon| \times \frac{M_{S C}}{m_{t}}$

We will find $\left|W_{d}\right| / m_{t}$ to be a function of just ambient conditions. Greater capture efficiency in (3) thus requiring lower $|\triangle s|$, an optimum value corresponds to eccentricity after capture $e_{\varepsilon}$ being just below unity. This allows to calculate efficiency from drag work along the parabolic orbit at periapsis. Using (2) and (3), we finally find an expression for capture efficiency, depending on Lorentz drag work and the hyperbolic velocity $v_{\infty \infty}$

$\frac{\left|W_{d}\right|}{m_{t} v_{s o}^{2} / 2}=\frac{M_{S C}}{m_{t}} \times \frac{e_{t}-e_{c}}{e_{h}-1} \sim \frac{M_{S C}}{m_{t}}$

We may calculate drag work using the radial speed rate in parabolic orbits to witite

$\int_{\Delta t}\left|\dot{W}_{d}\right| \mathrm{d} t=2 \times \int_{r_{p}}^{r_{u}} \frac{\left|\dot{W}_{d}\right| \mathrm{d} r}{\mathrm{~d} r / \mathrm{d} t}=2 \int_{r_{p}}^{r_{p}} \frac{\left|\dot{W}_{d}\right| r \mathrm{~d} r}{\sqrt{2 \mu\left(r-r_{p}\right)}}$

with $r_{i}$ a yet undefined upper limit. Next we calculate drag power

$\hat{W}_{d}=\mathbf{v} \cdot\left[L_{t} \mathbf{I}_{a v} \wedge \mathbf{B}\right]=-L_{t} \mathbf{I}_{d v} \cdot(\mathbf{v} \wedge \mathbf{B})$

using length-averaged current $I_{u v}$ as bounded by the short-circuit value, $\sigma_{t} E_{m} \times h_{t} w_{t}$, with $\sigma_{t}, h_{t}$ and $w_{t}$ being tape-tether conductivity, thickness and width,
$L_{t} \mathbf{I}_{a v}=L_{t} \times i_{a n^{\prime}} w_{t} h_{t} \sigma_{f} E_{m} \times \mathbf{u}_{f}, \quad i_{\mathfrak{a n}^{\prime}}\left(L_{t} / L_{\star}\right)<1$

$L_{\star} \propto\left(E_{M} / N_{e}^{2}\right)^{1 / 3}$ being certain characteristic ambient-plasma length, $I_{a v}$ only depending on electron density through $i_{s v}$ [9]. With the tether along the motional field $\mathbf{E}_{m}$, drag power in (6) becomes

$\dot{W}_{d}=-i_{a r^{\prime}} \sigma_{t} L_{t} w_{t} h_{t} \times\left(\mathbf{v}^{\prime} \wedge \mathbf{B}\right) \cdot(\mathbf{v} \wedge \mathbf{B})=-i_{a v} \sigma_{t} \frac{m_{t}}{P_{t}} B^{2}\left(\mathbf{v} \cdot \mathbf{v}^{\prime}\right)$

and, using conservation of angular momentum, we write

$\mathbf{v} \cdot \mathbf{v}^{\prime}=\mathbf{v} \cdot\left(\mathbf{v}-\Omega r \mathbf{u}_{e}\right)=v^{2}-\Omega r_{p} v_{p} \Rightarrow$

$r\left(\mathbf{v} \cdot \mathbf{v}^{\prime}\right)=r\left[v^{2}-\Omega r_{p} v_{p}\right]=2 \mu\left[1-r \Omega \frac{r_{p}}{2 \mu} \sqrt{\frac{2 \mu}{r_{p}}}\right]=2 \mu\left(1-\frac{r}{r_{M}}\right)$

$r_{M} / r_{p} \equiv \sqrt{2}\left(a_{S} / r_{p}\right)^{3 / 2} \propto\left(\rho_{p} / \Omega^{2}\right)^{1 / 2}$

with $r_{M}$, where $\mathbf{v} \cdot \mathbf{v}^{\prime}$ changes sign, being the maximum radius presenting drag, and $a_{S}=\left(\mu / \Omega^{2}\right)^{1 / 3}$ being the radius of an equatorial circular orbit where velocities of $S / C$ and co-rotating plasma are equal, $\left(\mu / a_{s}\right)^{1 / 2}=\Omega a_{S}$. The last expression above applies in the present analysis, where $r_{p} \approx R$.

We shall use values at $a_{S}$ as reference values, introducing the magnetic dipole law to wtite $B(r)=B_{S} a_{S}^{3} / r^{3}$, finally writing Eq. (4) as

$\left|W_{d}\right| / \frac{1}{2} m_{t} v_{\infty}^{2} \equiv \tilde{B}_{S}^{2} \times \hat{W}$

$\tilde{B}_{S}^{2}=\sigma_{i} B_{S}^{2} a_{S} v_{S} / 2^{5 / 6} p_{l} v_{\infty}^{2}$

$\dot{W}=2<i_{u v}>_{r} \times \dot{r}_{M}^{g / 3} \int_{1}^{\bar{P}_{M}} \frac{\left(\dot{r}_{M}-\tilde{r}\right) \mathrm{d} \dot{r}}{\hat{r}^{6} \sqrt{\tilde{r}-1}}, \quad\left(\dot{r} \equiv r / r_{p}\right)$

As regards the above radial-average of the notmalized lengthaverage current $i_{a b}$, consider the particularly simple regime for $L_{t} / L_{\star}>4$

$1-i_{a v}=\frac{L_{\star}}{L_{t}}\left[\propto \frac{E_{i n}^{1 / 3}}{L_{t}} \times\left(\frac{\sigma_{t} h_{t}}{N_{\varepsilon}}\right)^{2 / 3}\right], \quad \frac{L_{\star}}{L_{t}}<\frac{1}{4}$

yielding $i_{\mathrm{fl}}>0.75$ [9]. If this regime applies at the lowest relevant $N_{e}$ values, it should allow writing $\left\langle i_{a v}\right\rangle_{r} \approx 1$. However small plasma density $N_{\varepsilon}$ might reasonably be, it may be balanced by using a long enough tether. Note that this would favour using tethers for heavier $S$ / $C$. Actually, a longer tether may keep the same mass by reducing width, condition for validity of the standard OML (orbital motion limited) regime on current collection by a tether, is keeping $w_{t}$ below some upper bound, roughly 4 times Plasma Debye length.

Note now the very steep decrease away from periapsis in the radial rate of (14), as advanced,

$1 / \tilde{r}^{6} \sqrt{\check{r}-1}$

calculations of the integral not requiring to go far from $\vec{r}=1$. Independently, $r_{M}$ places a definite limit to the range of integration. Which limitation is more effective, depends on the $M$ value. For a large value, case of Neptune, as shown in Table 1, one can just ignore $\hat{r}$ in the numerator inside the integral, altogether.

On the other hand, for a smaller $R_{M}$ as in the case of Saturn, it pays

Table 1

Saturn/Neptun comparison.

\begin{tabular}{lll}
\hline & Saturn & Neptune \\
\hline$f_{p t}\left(\mathrm{gr} / \mathrm{cm}^{3}\right)$ & 0.6871 & 1.638 \\
Period (hours) & 10.656 & 16.11 \\
Drag reach $r_{M}$ (in parabolic orbit) & $3.7 R_{S}$ & $8.9 R_{N}$ \\
Dipole Tilt & $<0.5^{\circ}$ & $47^{\circ}$ \\
Dipole Offset & $0.06 R_{S}$ & $0.55 R_{N}$ \\
\hline
\end{tabular}




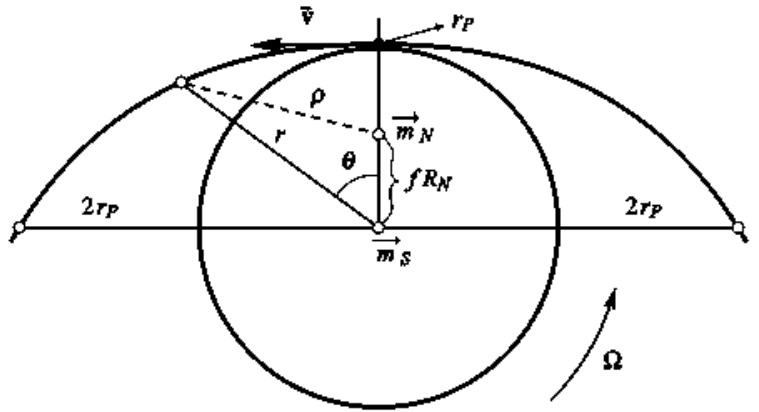

Fig. 1. Position of the magnetic dipole and approximate $S C$ trajectory.

to eliminate its limiting chatacter by moving from prograde to retrograde orbiting, just wtiting $r_{M}+r$ in that numerator, with a gain in the integral (and in capture efficiency) of about 2 . The integral converges very rapidly, increasing from $0.971 \hat{r}_{M}$ to just $1.008 \hat{r}_{M}$, if taking the upper limit from 1.5 to 2 [6]. In the present work we will go back to prograde capture, the drop by half in capture efficiency not being determinant here; our goal is allowing slow flybys exploration of moon Enceladus. Table 1 clearly shows that Saturn and Neptune present opposite cases among the Outer Giant Planets as regards density and spin, as emerging from the last expression in (11), resulting in opposite extremes in radial reach of Lorentz drag, $3.7 R_{S}$ as against $8.9 R_{N}$.

Another basic difference among Outer Giants arises from the magnetic structure of the field $\mathbf{B}$. A planetary magnetic field is due to a system of stationary currents in small volumes inside, as described by its magnetic moment and dipole law. The Saturn dipole is located at its center and parallel to the rotation axis; this holds approximately for Jupiter, too. On the other hand, the Neptune dipole is highly tilted and offset. For Neptune capture, efficiency depends on the longitude at periapsis; Fig. 1 shows the optimum case, with the $S / C$ facing the dipole when reaching petiapsis, with minimum distance $\rho$ to the dipole. Lorentz drag decreasing with the inverse $6^{\text {th }}$ power of $p$, faster convergence in the capture work integral allows not to go beyond about $1.12 r_{p}$ [10], as against $1.5 r_{p}$ for Saturn. Both basic differences between extreme Saturn and Neptune cases, suggest that joint tether missions to different planetary systems of moons might not be effective.

There is a further condition that separates Neptune of the other 3 Outer Giants. It has just 1 large moon, Triton, which is in retrograde orbit. Although unnecessary for greater capture efficiency because of the large $8.9 R_{N}$ value, visiting that moon will certainly need retrograde capture. Along with the final Outer planet, Uranus, having its rotation axis nearly lying in the ecliptic plane, both singular differences might be signs of collisions with other big bodies in the intriguing, early Solar System dynamics.

\subsection{Moon/spacecraft resonances}

Following capture, the $S / C$ apoapsis can be lowered, at given periapsis, to a circular moon orbit, allowing for a $1: 2$ resonance with the $S$ / $C$ elliptical orbit if the moon orbital radius satisfies certain condition. This would allow conveniently slow, tangential $S / C$ flybys of the moon, with two periapsis passes for each flyby. In the present problem the periapsis value was fixed, $r_{p} \approx R$, to make capture efficient, as we argued at length above. We have just found that moon Enceladus does satisfy the condition for that $1: 2$ resonance:

The period of a moon circular orbit of radius $r_{m}$, around a planet with gravitational constant $\mu$, is $\tau_{m}=2 \pi r_{m}^{3 / 2} / \sqrt{\mu}$. The period of an elliptical orbit with given periapsis $r_{p}$, and apoapsis at $r_{m}$, eccentricity being $e_{S C}=\left(r_{i n}-r_{p}\right) /\left(r_{m}+r_{p}\right)$, is

$\tau_{S C}=\frac{2 \pi}{\sqrt{\mu}}\left(\frac{r_{p}}{1-e_{S C}}\right)^{3 / 2}=\frac{2 \pi}{\sqrt{\mu}}\left(\frac{r_{m}}{1+e_{S C}}\right)^{3 / 2}$
Condition for $1: 2$ resonance being $\tau_{m}=2 \tau_{S c}$, there results $e_{S C}=4^{1 / 3}-1 \approx 0.59$, and

$\frac{r_{F}}{r_{m}}(\approx 0.2597)=2^{1 / 3}-1(\approx 0.2599)$

where we used values $r_{i n}=r_{E n c} \approx 238000 \mathrm{~km}$, and $r_{p} \approx R_{S}+$ capture - periapsis altitude $\approx 60300 \mathrm{~km}+1500 \mathrm{~km}$ for some representative altitude, affecting little the $r_{p} / r_{\text {in }}$ ratio.

Certainly, the $1: 2$ resonance does not apply to moon Dione, for which we will use non-tangential flybys at 1:1 resonance, with one periapsis pass per flyby. Now we will have $\tau_{S C}=\tau_{D}$, i.e.,

$\frac{2 \pi}{\sqrt{\mu}}\left(\frac{r_{p}}{1-e_{S C}}\right)^{3 / 2}=\frac{2 \pi}{\sqrt{\mu}} r_{D}^{3 / 2}$

yielding $1-e_{S C}=r_{F} / r_{D} \approx 0.160$, having used $r_{D} \approx 377400 \mathrm{~km}$ and $r_{p} \approx R_{S}$. We thus find $e_{S C} \approx 0.840$, with the apoapsis of the orbit at $1: 1$ resonance with Dione, $r_{o}=\left(1+e_{S C}\right) \times r_{D} \approx 694400 \mathrm{~km}$, lying between moons Rhea and Titan. Note that 1 revolution period of moon Dione (2.74 days) does correspond to 1 periapsis pass of the $S / C$ during Dione flybys, whereas it corresponds to $4 S / C$ periapsis passes ( 0.685 days) during the Enceladus flybys, when Dione, Enceladus, and the spacecraft are in 1:2:4 resonance.

Apoapsis lowering will occur at successive periapsis passes, resulting in a series of elliptical orbits with common periapsis and decreasing eccentricities, with changes in periapsis position being small second-order effects. As noticed in the following section, eccentricity decrements being small, about 0.02 say, like at capture itself, calculations may be carried out as if eccentricity, though different from unity, was kept constant during each perijove pass. Changes in calculation, as compared with the capture analysis, are using the energy equation for each patticular eccentricity value,

$v^{2}=\mu\left(\frac{2}{r}-\frac{1-e}{r_{p}}\right)$

instead of $v^{2}=2 \mu / r$, while using the radial speed rate $\mathrm{d} r / \mathrm{d} t$ value resulting from (20) and the angular-momentum conservation law,

$r^{2} \mathrm{~d} \theta / \mathrm{d} t=r_{p} v_{p e}$

We then have

$\left(\frac{\mathrm{d} r}{\mathrm{~d} t}\right)^{2}=v^{2}-\left(r \frac{\mathrm{d} \theta}{\mathrm{d} t}\right)^{2}=\mu\left(\frac{2}{r}-\frac{1-e}{r_{p}}\right)-\frac{(1+e) \mu r_{p}}{r^{2}}$

where we used the periapsis velocity $v_{p e}=\left[\mu(1+e) / r_{p}\right]^{1 / 2}$. Radial speed-rate vanishing, in (22) above, occurs at 2 values of tadius, periapsis $r_{p}$ and apoapsis $r_{\alpha}=r_{p}(1+e) /(1-e)$, finally yielding

$\frac{\mathrm{d} r}{\mathrm{~d} t}=\sqrt{(1+e) \mu\left(r-r_{p}\right)\left[1-\left(r / r_{a}\right)\right]} / r$

replacing the radial rate at $e=1$, when the apoapsis radius $r_{u}=r_{p}(1+e) /(1-e)$ was at infinity.

Next, use (20) in Eq. (9), and write

$$
\begin{array}{r}
r \mathbf{v} \cdot \mathbf{v}^{\prime}=r\left[\mu\left(\frac{2}{r}-\frac{1-\varepsilon}{r_{p}}\right)\right]-r \Omega r_{p} v_{p k^{*}}=2 \\
\mu\left[1-\frac{r}{r_{p}} \frac{1-\varepsilon}{2}-r \frac{\Omega r_{p}}{2 \mu} \sqrt{\frac{\mu(1+\varepsilon)}{r_{p}}}\right] \\
r \mathbf{v} \cdot \mathbf{v}^{\prime}=2 \mu\left[1-r\left(\frac{1-\varepsilon}{2 r_{p}}+\sqrt{\frac{1+\varepsilon}{2}} \frac{1}{r_{M}}\right)\right]
\end{array}
$$

replacing $2 \mu\left[1-\left(r / r_{M}\right)\right]$, from Eq. (10), for $e=1$.

\section{A Dione-Enceladus tour scheme}

Using the above results for arbitrary eccentricity $\ell$, yields $\left|W_{d e}\right| /\left(m_{t} v_{\infty}^{2} / 2\right)=\hat{B}_{S}^{2} \hat{W}_{c}$ 
Table 2

Values of the function $f\left(\tilde{r}, \tilde{r}_{M}, e\right)$ (Eq. (26)).

\begin{tabular}{llll}
\hline$\tilde{r}$ & $e=1$ & $e=0.84$ (Differences) & $e=0.59$ (Differences) \\
\hline 1 & 2.70 & $2.66(1.5 \%)$ & $2.67(1.1 \%)$ \\
1.25 & 2.45 & $2.35(4.3 \%)$ & $2.23(9.9 \%)$ \\
1.50 & 2.20 & $2.03(8.4 \%)$ & $1.75(25.7 \%)$ \\
\hline
\end{tabular}

$\tilde{W}_{e}=2<i_{a v}>_{r} \tilde{r}_{M}^{8 / 3} \int_{1}^{\tilde{u}_{u}} \frac{\mathrm{d} \tilde{r}}{\tilde{r}^{6} \sqrt{\tilde{r}-1}} \times f\left(\tilde{r}, \tilde{r}_{M}, e\right)$

$f\left(\tilde{r}, \tilde{r}_{M}, e\right)=\frac{\tilde{r}_{M}-\tilde{r}\left[\sqrt{(1+e) / 2}+\tilde{r}_{M}(1-e) / 2\right]}{\sqrt{[(1+e)-(1-e) \tilde{r}] / 2}}$

which recovers Eq. (14) for $e=1$. Both $r_{M}$ and $\tilde{B}_{S}^{2}$ are as for $e=1$.

As $e$ is decreased from 1 , the upper limit in the integral keeps being the $r$-value for vanishing numerator in the second ratio above, until a value $e \approx 0.28$ is reached, with denominator vanishing too. At lower $e$, the upper limit in the integral is the apoapsis radius, at vanishing denominator. Note that this change in behavior occurs well below the $0.84<e<1$ range involved in reaching resonance with Dione and its flybys exploration, and below the $0.59<e<0.84$ range required for the Enceladus flybys.

An approximate, extremely simplified analysis is here discussed. For the eccentricity ranges of interest, $0.84<e<1$ and $0.59<e<0.84$, and the radial range of integration, $1<r / r_{p}<1.5$, Table 2 suggests that the functional factor $f\left(\tilde{r}, \tilde{r}_{M}, e\right)$ in Eqs. 25 and 26 might be reasonably approximated throughout by using its $e=1$ values, with $(25,26)$ just replaced by (14). The error would appear substantial, however, near the radial end, at the lower eccentricity. Let us recall, however, the very steep decrease away from periapsis, in the radial rate, given by (16), at both Eqs. (14) and (25), making values near $r=1.5 r_{p}$ contribute little to integrals involving that rate, as noticed in the paragraph following Eq. (16). Results from the simplified analysis might reasonably apply for the $0.59<e<1$ tour.

\subsection{Moon tour}

With Eq. (14) replacing $(25,26)$ in the above simplified analysis, $S$ / $C$-orbit evolution can be described in terms of number of successive periapsis passes. For $M_{S C} / m_{t}$ such that $e_{\mathcal{c}}$ is about 0.99 , it would take 7 steps following capture, for the $S / C$ to reach $e=0.85$, with the capture value $\Delta e \approx 0.02$. A last eccentricity decrement must be reached in two convenient steps by switching the current off appropriately over part of the drag arc to allow for a first flyby of Dione while reducing $\Delta e$ by half. Switching off the current afterward over the entire resonance orbit would allow for repeated flybys, with the moon overtaking each time the $S / C$. It would later take 13 steps to reach Enceladus at $e=0.59$ from Dione at $e=0.84$.

\subsection{Power generation}

A large amount of energy could be taken from the orbital motion of the $S / C$ during capture and during each one of the successive periapsis passes, into the tether electric circuit. A fraction taken by electric loads at the cathodic end, could have a weak effect on tether current and the related tether dynamics. A part of that energy could be used during each of the operation steps, with the rest saved/stored in batteries, for later use. Operation would keep emphasis on reserving power for use at the planned steps at Enceladus, main objective of the mission, as regards life search.

The revolution period of Dione is about 2.74 days. The $S / C$ at resonance $1: 1$ would take 13.70 days in making, say, 5 flybys. The $S / C$ at resonance $1: 2$ would take 1 flyby per period of Enceladus, or 13.70 days in making 10 flybys.

\subsection{Minor issues}

Radiation is a big issue for tethers at Jupiter but not at Saturn, which lacks strong radiation belts. As regards tether dynamic instabilities, they are generally quite slow [11], while the times involved in the mission are short. Regarding the $J_{2}$ zonal harmonic coefficient, which is 15 times as large as Earth's, and comparable to Jupiter, it requires a minor correction involving apsidal precession, the way it was made for Jupiter [5], to consider in planning an Enceladus-Dione mission.

\section{Summary of results}

Saturn moon Enceladus stands out, along with Jovian moon Europa, as particular moon of interest in the search for conditions of habitability, in parallel to search for life, in the ice moons of the Outer Giant Planets. Moons Enceladus and Dione are in 1:2 resonance in the Saturn system. The very recent discovery (fall of 2018), as result of mission Cassini, of intriguing linear virgae at Dione, makes exploring both moons a convenience. We have presented a tether-mission concept making possible multiple flybys of both moons, though Enceladus would be of most critical interest. We have proved that a $S C$ orbit at Saturn with periapsis very near planet radius as required by a tether, and apoapsis at Enceladus orbit satisfies the condition for a $1: 2 \mathrm{moon} / S C$ resonance, allowing conveniently slow, parallel SC flybys of the moon. Our analysis shows that following magnetic capture by the SC-tether, repeated free Lorentz-drag, keeping periapsis near constant, could take the SC apoapsis down to Enceladus.

\section{Acknowledgements}

This work was supported by MINECO/AEI, under Project ESP201787271-P, and FEDER/EU.

\section{References}

[1] J.R. Spencer, F. Nimmo, Enceladus: an active ice world in the Saturn system, Annu. Rev. Earth Planet Sci. 41 (2013) 693-717, https://doi.org/10.1146/annurev-earth. 050212-124025.

[2] H.W. Hsu, F. Postberg, Y. Sekine, T. Shibuya, S. Kempf, M. Horányi, A. Juhász, N. Altobelli, K. Suzuki, Y. Masaki, et al., Ongoing hydrothermal activities within Enceladus, Nature 519 (7542) (2015) 207-210, https://doi.org/10.1038 nature14262.

[3] J.H. Waite, C.R. Glein, R.S. Perryman, B.D. Teolis, B.A. Magee, G. Miller, J. Grimes, M.E. Perry, K.E. Miller, A. Bouquet, et al., Cassini finds molecular hydrogen in the Enceladus plume: evidence for hydrothermal processes, Science 356 (6334) (2017) 155-159, https://doi.org/10.1126/science.aai8703.

[4] E.S. Martin, D.A. Patthoff, Mysterious linear features across Saturn's moon Dione, Geophys. Res. Lett. 45 (20) (2018) 10-978, https://doi.org/10.1029/ 2018 gl079819.

[5] J.R. Sanmartin, M. Charro, E.C. Lorenzini, H. Garrett, C. Bombardelli, C. Bramanti, Electrodynamic tether at Jupiter - II: fast moon tour after capture, IEEE Trans. Plasma Sci. 37 (4) (2009) 620 626, https://doi.org/10.1109/tps.2009.2013955.

[6] J.R. Sanmartín, J. Peláez, I. Carrera-Calvo, Comparative Saturn-versus-Jupiter tether operation, J. Geophys. Res.: Space Physics 123 (7) (2018) 6026-6030, https:// doi.org/10.1029/2018ja025574.

[7] J.R. Sanmartín, M. Charro, H.B. Garrett, G. Sánchez-Arriaga, A. Sánchez-Torres, Analysis of tether-mission concept for multiple flybys of moon Europa, J. Propuls. Power 33 (2) (2017) 338-342, https://doi.org/10.2514/1.B36205.

[8] R. Battin, An Introduction to the Mathematics and Methods of Astrodynamics Revised Edition, AIAA, ALAA education series, Reston, VA, 1999.

[9] J.R. Sanmartin, M. Charro, E.C. Lorenzini, H. Garrett, C. Bombardelli, C. Bramanti, Electrodynamic tether at Jupiter - I: capture operation and constraints, IEEE Trans. Plasma Sci. 36 (5) (2008) 2450-2458, https://doi.org/10.1109/TPS.2008. 2002580 .

[10] J.R. Sanmartín, J. Peláez, Tether capture of spacecraft at Neptune, Acta Astronaut (SI:Tethers in Space 2019) (2020) Submitted for publication.

[11] J. Pelaez, E. Lorenzini, O. Lopez-Rebollal, M. Ruiz, A new kind of dynamic in stability in electrodynamic tethers, J. Astronaut. Sci. 48 (2000) 449-476, https:// doi.org/10.2514/1.6473. 